\title{
Magneto-Convective Analyses of the PbLi Flow for the EU-WCLL Fusion Breeding Blanket
}

\author{
Fernando R. Urgorri *D, Ivan Fernández-Berceruelo (D) and David Rapisarda \\ Centre for Energy Environment and Technology Research, National Fusion Laboratory, 28040 Madrid, Spain; \\ ivan.fernandez@ciemat.es (I.F.-B.); david.rapisarda@ciemat.es (D.R.) \\ * Correspondence: fernando.roca@ciemat.es
}

Citation: Urgorri, F.R.; FernándezBerceruelo, I.; Rapisarda, D. MagnetoConvective Analyses of the PbLi Flow for the EU-WCLL Fusion Breeding Blanket. Energies 2021, 14, 6192. https://doi.org/10.3390/en14196192

Academic Editors: Marica Eboli and Dan Gabriel Cacuci

Received: 19 July 2021

Accepted: 21 September 2021

Published: 28 September 2021

Publisher's Note: MDPI stays neutral with regard to jurisdictional claims in published maps and institutional affiliations.

Copyright: (c) 2021 by the authors. Licensee MDPI, Basel, Switzerland. This article is an open access article distributed under the terms and conditions of the Creative Commons Attribution (CC BY) license (https:/ / creativecommons.org/licenses/by/ $4.0 /)$.

\begin{abstract}
The Water Cooled Lithium Lead (WCLL) breeding blanket is one of the driver blanket concepts under development for the European Demonstration Reactor (DEMO). The majority of the blanket volume is occupied by flowing $\mathrm{PbLi}$ at eutectic composition. This liquid metal flow is subdued to high fluxes of particles coming from the plasma which are translated into a high non-homogeneous heat volumetric source inside the fluid. The heat is removed from the PbLi thanks to several water tubes immersed in the metal. The dynamics of the PbLi is heavily affected by the heat source and by the position of the tubes. Moreover, the conducting fluid is electrically coupled with the intense magnetic field used for the plasma confinement. As a result, the PbLi flow is strongly affected by the Magnetohydrodynamics (MHD) forces. In the WCLL, the MHD and convective interactions are expected to be comparable. Therefore, the PbLi dynamics and consequently the heat transfer between the liquid metal and the water coolant will be ruled by the magneto-convective phenomenon. This work presents 3D computational analyses of the PbLi flow in the frontal region of the WCLL design. The simulations include the combined effect of MHD forces caused by the magnetic field and the buoyancy interaction created by the temperature distribution. The latter is determined by the PbLi dynamics, the volumetric heat source and the position of the water tubes. Simulations have allowed computing the heat transfer between the PbLi and the water tubes. Nusselt and Grashof numbers have been obtained in the different regions of the system.
\end{abstract}

Keywords: magneto-convection; Magnetohydrodynamics; heat transfer; WCLL

\section{Introduction}

Breeding blankets are crucial systems projected in future nuclear fusion reactors by magnetic confinement whose main purpose is to regenerate the tritium burnt in the plasma. They are designed to absorb the energetic neutrons created in the fusion reactions $\left(D+T \rightarrow^{4} \mathrm{He}+n\right)$. In the core of the blanket, the neutrons produce tritium by transmuting the lithium contained in the blanket. The amount of tritium bred this way has to be extracted from the reactor for being processed, stored and eventually injected in the plasma. When including a neutron multiplier in the blanket, the tritium bred in the blanket can be higher than the tritium burnt in the plasma which allows maintaining the desired tritium self-sufficiency of the plant.

Within the framework of the EUROfusion blanket project [1,2], the WCLL blanket concept [3] has been selected as one of the two candidates for driver blanket of the European DEMO. This concept is based on an eutectic alloy of $\mathrm{PbLi}$ ( $\mathrm{Pb}$ acting as neutron multiplier and Li acting as breeder) flowing at low velocities. The blanket is directly exposed to highly energetic particle fluxes coming from the blanket. For this reason, liquid water pressurized at 155 bar is used for cooling both the PbLi and the steel structures. A network of water tubes immersed in the liquid metal is included in the design for this purpose.

The PbLi is a good electrical conductor immersed in the strong magnetic field used for the plasma confinement. Therefore, electric currents will be induced inside the flow 
producing Lorentz forces that alter its dynamics. This interaction known as Magnetohydrodynamics (MHD) is dominant in most of the WCLL PbLi flowpath. Nevertheless, in the regions closer to the first wall (FW), the high particle fluxes from the plasma are very energetic which are translated into a very high non-homogeneous heat source. This together with the arrangement of the cold water tubes immersed in the PbLi flow can produce temperature differences inside the bulk of the fluid of hundreds of degrees. In these areas, the buoyancy forces are expected to be comparable or even higher than the Lorentz ones.

In this work, magneto-convective simulations of the WCLL blanket are presented. Those are focused on a very particular region of the design: the frontal part of the central outboard elementary cell (Figure 1). This region is located very close to the plasma and it is characterized by a very high heat flux and a consequent dense arrangement of water tubes. This location is of the special interest from the blanket design perspective since it presents a very high tritium production. In its vicinity, it is expected that buoyancy forces play a very important role on PbLi dynamics.

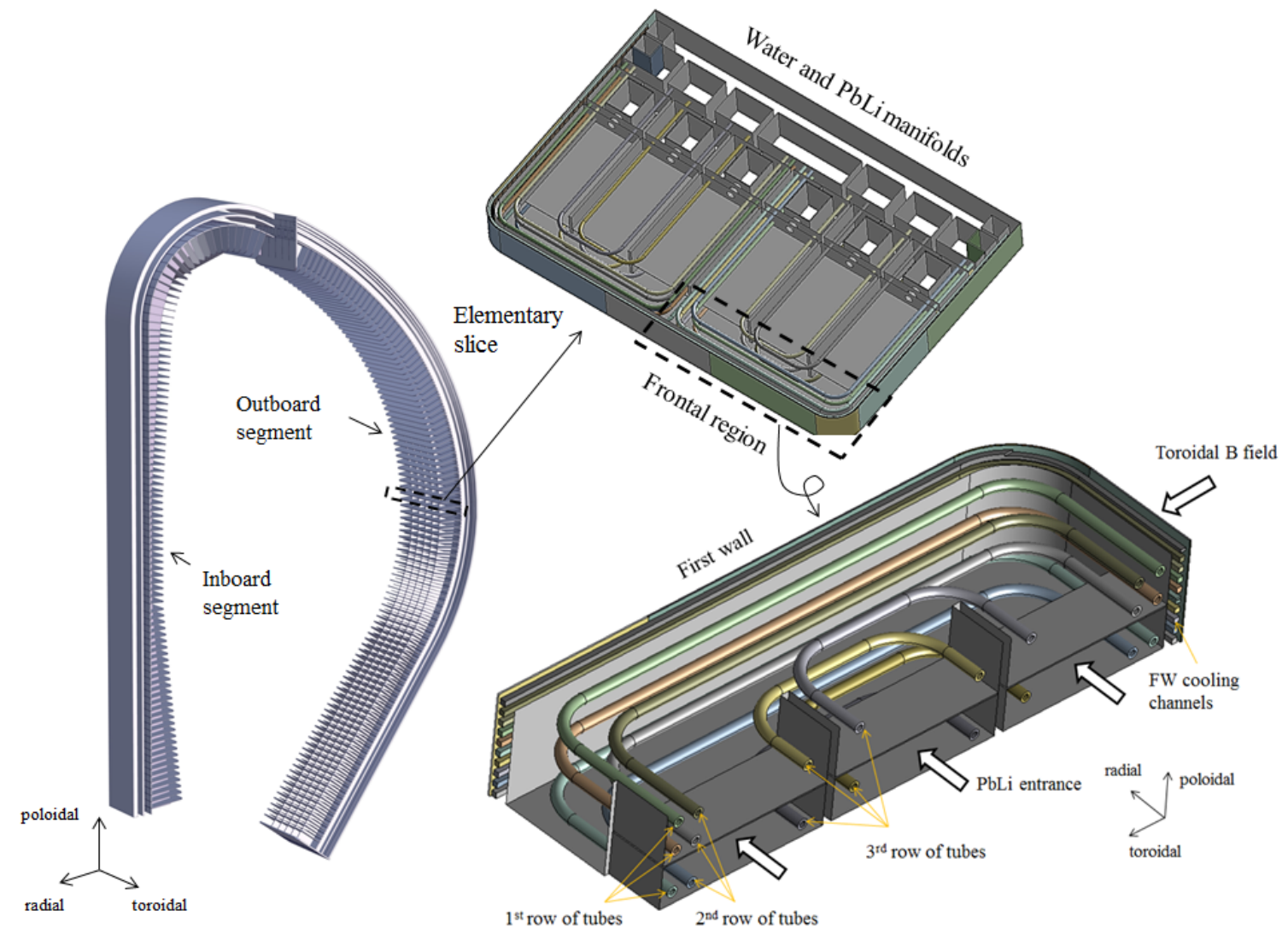

Figure 1. Detailed view of the WCLL blanket design: WCLL segments (left), elementary cell (top-right), frontal region (bottom-right).

The WCLL elementary cell is composed of six parallel circuits along the toroidal direction. Each of them is fed by a rear PbLi manifold and presents a U-shape flowpath along the radial direction. The analyses of this work are focused on the frontal part of one of the six parallel circuits; in the area where the flow describes a $180^{\circ}$ turn, close to the FW of the blanket. It is worth noting that there is not a physical separation in between the frontal regions of the six parallel circuits. Therefore, in a central circuit, such as the one considered in this analysis, the lateral radial-poloidal walls only cover the rear part of the domain (the so-called radial channels Figure 2). 


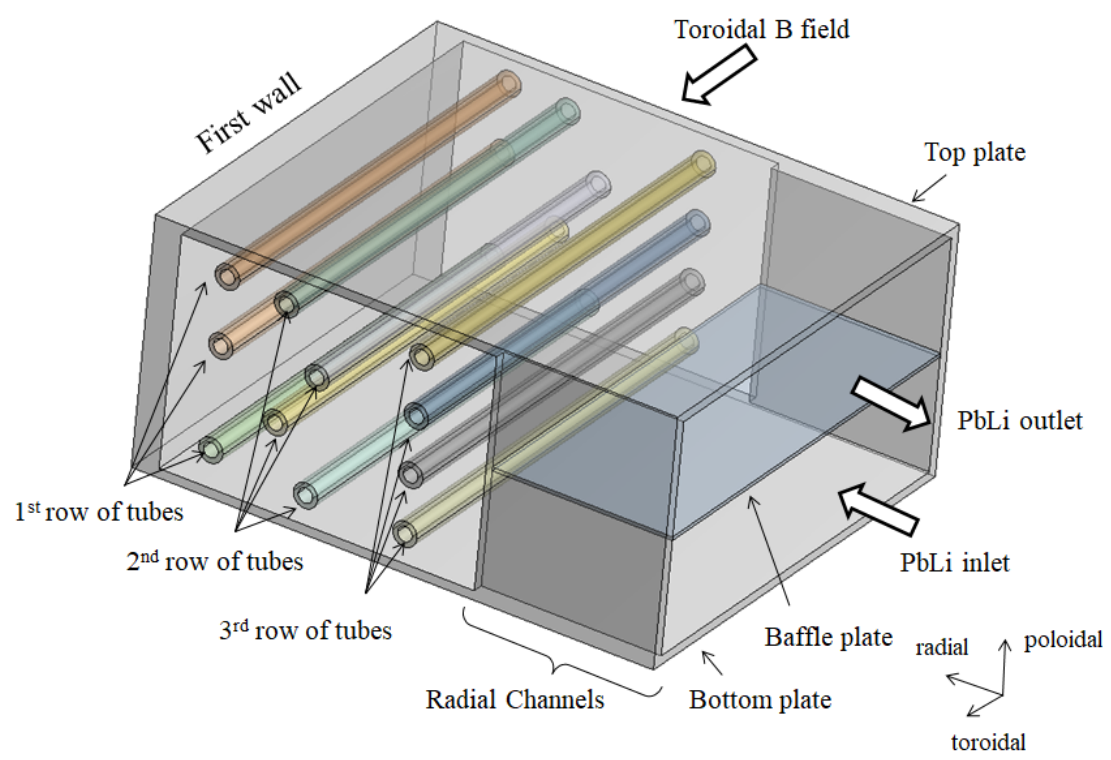

Figure 2. Geometry considered in the magneto-convective simulations.

The numerically analyzed geometry has been simplified. In particular, the shape of the third row of tubes has been straightened, making them completely toroidaly oriented. This way, the studied geometry presents a good symmetry along the toroidal direction which allows using structured meshes in the analyses. Indeed, structured meshes provide better stability in MHD simulations. In the ANSYS-Fluent MHD solver, unstructured meshes can lead to violations of charge conservation forcing extremely small time steps which would rise the computational cost.

The simplification performed does not affect the first and second rows of tubes which have the same shape and position than in the WCLL design. These tubes are closer to the FW where buoyancy forces are expected to be stronger. The square section FW cooling channels are not directly included in the computational domain. It only covers the part of the FW in between the cooling channels and the PbLi.

\section{Numerical Model}

In the present analyses, the dynamics of the PbLi flow is ruled by the Navier-Stokes equation in which the Lorentz and buoyancy terms are added as a volumetric sources:

$$
\rho_{0}\left(\frac{\partial \vec{u}}{\partial t}+(\vec{u} \cdot \nabla) \vec{u}\right)=\eta \nabla^{2} \vec{u}-\nabla p+\vec{j} \times \vec{B}_{0}+\rho(T) \vec{g}
$$

where the PbLi density and dynamic viscosity are denoted by $\rho$ and $\eta$, respectively. The vector field $\vec{u}$ is the velocity of the fluid. The Lorentz force is the cross product of the current density $(\vec{j})$ and the external magnetic field $\left(\vec{B}_{0}\right)$ which is assumed to be constant. $\vec{g}$ represents the gravity vector field and $T$ the temperature field.

Under the inductionless approximation (the magnetic field created by the induced currents is negligible in comparison with the external one), the electric potential $(\phi)$ is well defined and the generalized Ohm's law can be written as follows:

$$
\vec{j}=\sigma\left(-\nabla \phi+\vec{u} \times \overrightarrow{B_{0}}\right)
$$

where $\sigma$ is the electrical conductivity of the media. The MHD problem can be closed by applying the divergence to (2) and assuming charge conservation $(\nabla \vec{j}=0)$. Therefore, a Poisson's equation for the electric potential has to be solved coupled with (1). This equation is solved also in the solid domains. The electric potential and electric currents are continuous across the solid-fluid interfaces. 
Concerning the buoyancy forces in the fluid, they are treated using the Boussinesq approximation:

$$
\rho(T) \sim \rho_{0}\left(1-\beta\left(T-T_{0}\right)\right)
$$

where $T_{0}$ is the characteristic temperature of the problem, $\rho_{0}$ is the PbLi density at that temperature and $\beta$ is the thermal expansion coefficient. The rest of the fluid and solid properties are assumed to be constant.

The temperature field evolves following the energy conservation equation:

$$
\rho_{0} c_{p}\left(\frac{\partial T}{\partial t}+u^{j} \partial_{j} T\right)=\kappa \partial_{j} \partial^{j} T+Q
$$

where $c_{p}$ is the specific heat capacity of the material and $\kappa$ its thermal conductivity. The volumetric heat source $Q$ represents the effect of the neutrons and photons (nuclear heating). As a first approximation, this source only depends on the radial coordinate. The shape of this kind of functions are derived from the neutronics analyses performed for the WCLL (e.g., [4]). In this work, the following piecewise functions have been used. These functions provide a steeper power density in the region close to the FW in comparison with the exponential functions used in previous studies which are only accurate in the tail of the curve (e.g., [5,6]).

$$
\left.\begin{array}{c}
Q(r)_{P b L i}\left[\frac{\mathrm{MW}}{\mathrm{m}^{3}}\right]=\left\{\begin{array}{cc}
98.962 r+9.5968 & r>-0.05 \\
365.35 r^{4}+44.3 r^{3}+215 r^{2}+54.376 r+6.8797 & r<-0.05
\end{array}\right\} \\
Q(r)_{\text {steel }}\left[\frac{\mathrm{MW}}{\mathrm{m}^{3}}\right]=\left\{\begin{array}{cc}
9185.7 r-221.25 & r>-0.025 \\
483.1 r^{3}+576.96 r^{2}+85.61 r+5.88 & -0.025>r>-0.075 \\
253.5 r^{4}+296.81 r^{3}+142.72 r^{2}+35.2 r+3.95 & r<-0.075
\end{array}\right.
\end{array}\right\}
$$

The origin of the radial coordinate is located in the contact plane between the FW and the PbLi. The $r$ coordinate decreases towards the PbLi inlet and grows towards the FW. The shape of the volumetric heat source is plotted in Figure 3.

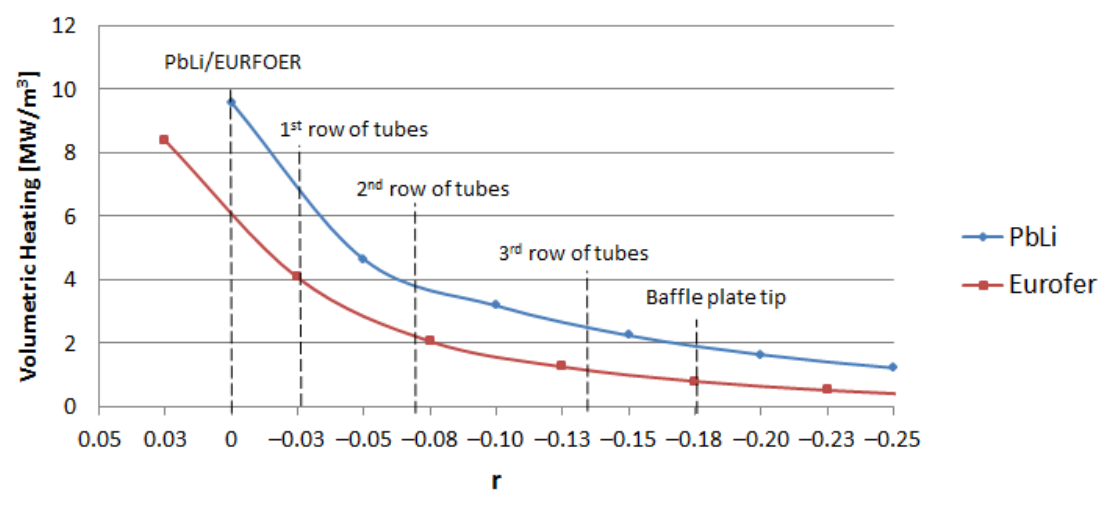

Figure 3. Volumetric heat source used in the computations.

The magnitude of the nuclear heating is of the order of $\mathrm{MW} / \mathrm{m}^{3}$. The Ohmic or Joule heating $\left(\sim j^{2} / \sigma\right)$ is expected to be several orders of magnitude smaller than the nuclear heating. Indeed, in the WCLL conditions: $u \sim \mathrm{mm} / \mathrm{s}, B_{0} \sim T$ and $\sigma \sim 10^{5}(\Omega \cdot \mathrm{m})^{-1}$, which means that: $j \sim \sigma u B_{0} \sim 10^{2} \mathrm{~A} / \mathrm{m}^{2}$. Thus, the Joule heating $\left(\sim 10^{-1} \mathrm{~W} / \mathrm{m}^{3}\right)$ has been neglected in the present analysis.

In a dimensionless analysis of (1), the square of the Hartmann number $(\mathrm{Ha})$ represents the ratio between Lorentz forces and Viscous forces:

$$
H a:=a B_{0} \sqrt{\frac{\sigma}{\eta}}
$$


where $a$ is the channel semilength along the field direction (toroidal direction). In the central outboard WCLL conditions, the Hartmann number in the frontal part of the blanket is estimated to be approximately 8800 . This means that viscous forces will be strongly suppressed by the magnetic field.

Complementary, the Grashof number $(G r)$ represents the ratio between the buoyancy and viscous forces:

$$
G r:=\frac{g \beta \Delta T b^{3} \rho^{2}}{\eta^{2}}
$$

where $b$ is the characteristic length of the heat transfer process, $\Delta T$ is the characteristic temperature difference and $g$ is the gravitational acceleration. Estimating $G r$ in the different regions of the WCLL is not a straightforward task. Indeed, the heat source is extended along the complete computational domain (in a non-homogeneous way) and there are multiple cooling regions. Therefore, both the characteristic length and the temperature difference are not known before performing an specific thermal calculation. It is sometimes found in the literature (e.g., $[7,8]$ ) an estimation of the characteristic temperature as $\Delta T=\frac{\bar{Q} b^{2}}{\kappa}$, using the semilength along the radial direction as the characteristic length. In the case of study, this strategy would provide an unrealistic temperature difference of several thousands of degrees and $G r \sim 10^{12}$ or more. This assumption is only valid (although very conservative) when the cooling comes from the sides of the system (e.g., a cooling plate of the Dual Coolant Lithium Lead blanket (DCLL)). Indeed, the expression does not take into account the cooling effect of the internal tubes which will keep temperatures differences in more moderate values, reducing $G r$ as well. More realistic estimations can be made with this formula considering only the PbLi volume contained in between two rows of tubes. With this strategy, $\Delta T \sim 10^{2} \mathrm{~K}$ and $\mathrm{Gr} \sim 10^{8}$.

In purely natural or free magneto-convection problems, buoyant forces are balanced with Lorentz forces. The characteristic velocity scale is given by the ratio between the $\mathrm{Gr}$ and $\mathrm{Ha}^{2}: U_{0}=\frac{\eta}{\rho_{0} L} \frac{G r}{H a^{2}}$ [9]. Alternatively, in mixed-convection problems, the velocity scale is given by the characteristic velocity scale of the pressure driven flow $\left(U_{0}\right)$. In these scenarios, the ratio between $\mathrm{Gr}$ and $\left(\mathrm{Re} \cdot \mathrm{Ha}^{2}\right)$ weights the relation between buoyant and Lorentz forces [10]. Indeed, normalizing $\vec{B}, \vec{u}, p, \vec{j}, \vec{g}$ and $T$ by $B_{0}, U_{0}, \sigma B_{0}^{2} L U_{0}, \sigma B_{0} U_{0},|\vec{g}|$ and $\Delta T_{0}$, respectively, Equation (1) can be written as follows:

$$
\frac{R e}{H a^{2}}\left(\frac{\partial \hat{u}}{\partial t}+(\hat{u} \cdot \nabla) \hat{u}\right)=\frac{1}{H a^{2}} \nabla^{2} \hat{u}-\nabla \hat{p}+\hat{j} \times \hat{B_{0}}+\frac{G r}{R e H a^{2}} \hat{T} \hat{g}
$$

where the symbol` represents a normalized variable (e.g., $\left.\hat{u}=\vec{u} / U_{0}\right)$. Inertial effects are weighted to the Lorentz forces with the usual interaction parameter $\left(N=\frac{H a^{2}}{R e}\right)$ or by the square of the so-called Lykoudis number $(L y)$ in natural convection problems: $L y^{2}=\frac{H a^{4}}{G r}$. In such problems, only for $\mathrm{Gr}$ of the order of $\mathrm{Ha}^{4}$, inertial effects are significant.

In natural convection situations, the Péclet number $(P e=R e \cdot P r)$ can be related also with $\mathrm{Gr}$ and $\mathrm{Ha}$ : $\mathrm{Pe}=\frac{\mathrm{Gr}}{\mathrm{H} a^{2}} \mathrm{Pr}$. Therefore, the advective heat transfer is strongly suppressed by the magnetic field. This effect is also true on mixed-convection problems although a sufficiently high $R e$ can as usual make advection a significant heat transfer mechanism.

Both natural and mixed-convection scenarios have been studied in the past for different combinations of $\mathrm{Ha}$, Re and $\mathrm{Gr}$ under the influence of a transversal magnetic field. MHD flows in vertical channels with a non-homogeneous heat source and insulated walls have been studied within the framework of the DCLL blanket [11,12]. Above a critical value of $\mathrm{Ha}$, the magnetic field is able to stabilize both the buoyancy-assisted (upward) flow [13] and buoyancy-opposed (downward) flow [14]. The latter requires more intense magnetic fields for the stabilization.

In the case of horizontal ducts, both natural convection [15] and mixed-convection scenarios [10] have been studied considering heated surfaces. Volumetric heat sources in horizontal channels have been studied for natural-convection regimes within the frame- 
work of the Helium Cooled Lithium Lead (HCLL) blanket concept analyses [16]. In this case, the cooling plates and the spatially varying heat source originate the flow movement. More recently, studies dedicated to the WCLL blanket show that the presence of the cooling tubes immersed in the $\mathrm{PbLi}$ also plays an important role on determining the buoyant recirculation patterns $[17,18]$.

The strong magnetic field and heat source present in the frontal part of the WCLL blanket implies a very challenging problem from the computational point of view. Being able to resolve the MHD boundary layers while capturing the dynamics of the buoyant vortexes requires very small mesh sizes and very small time steps in a complex geometry. To relax the computational requirements, this work considers reduced $\mathrm{Ha}$ values. The final objective would be to gradually increase $\mathrm{Ha}$ until reproducing the real blanket conditions. In this paper, two different situations are compared: $\mathrm{Ha}=1000$ and $\mathrm{Ha}=2000$. In each case, the external magnetic field has been tuned accordingly.

To keep the ratio between Lorentz and buoyant forces as similar as possible to the WCLL conditions, the gravity field has been reduced as well keeping the ratio between $\mathrm{Gr}$ and $\mathrm{Ha}^{2}$ constant. For this purpose, the reduced gravity field $\left(g_{r}\right)$ and reduced magnetic field $\left(B_{r}\right)$ follow the following scaling rule:

$$
\frac{g_{r}}{g_{0}}=\left(\frac{B_{r}}{B_{0}}\right)^{2}
$$

Equation (10) will only keep the ratio $\frac{\mathrm{Gr}}{\mathrm{Ha} \mathrm{C}^{2}}$ constant if the characteristic temperature difference of the reduced case $\left(\Delta T_{r}\right)$ is equal to real $\Delta T$. Since the heat source is the same, this is expected to be approximately true. Results obtained in Section 3 for different values of $B_{r}$ and $g_{r}$ are consistent with this approximation.

\section{Simulation Conditions}

The material properties of the PbLi [19] and the steel (EUROFER [20]) at $T_{0}=600 \mathrm{~K}$ are exposed in Table 1.

Table 1. PbLi and EUROFER properties at $T_{0}=600 \mathrm{~K}$.

\begin{tabular}{ccc}
\hline & PbLi & EUROFER \\
\hline$\rho\left(\mathrm{kg} / \mathrm{m}^{3}\right)$ & 9806 & 7674 \\
$c_{p}(\mathrm{~J} / \mathrm{kg} \cdot \mathrm{K})$ & 189.5 & 565 \\
$\mathcal{\kappa}(\mathrm{W} / \mathrm{m} \cdot \mathrm{K})$ & 20.93 & 29.74 \\
$\sigma\left(\Omega^{-1} \cdot \mathrm{m}^{-1}\right)$ & $7.82 \times 10^{5}$ & $1.07 \times 10^{6}$ \\
$\beta\left(\mathrm{K}^{-1}\right)$ & $1.21 \times 10^{-4}$ & - \\
$\eta(\mathrm{Pa} \cdot \mathrm{s})$ & $1.93 \times 10^{-3}$ & - \\
\hline
\end{tabular}

Additionally, the geometrical inputs taken from the WCLL frontal region are exposed in Figure 4. The toroidal length of the domain is $243 \mathrm{~mm}$.

Regarding the boundary conditions, convective ones $\left(q=h\left(T-T_{f}\right)\right)$ have been used in the internal surfaces of the tubes and in the external side of the FW to represent the cooling effect of the water. The water stream temperatures $\left(T_{f}\right)$ of both circuits are assumed at $311.5^{\circ} \mathrm{C}$. This value is the average temperature of the water thermal cycle [18]. The heat transfer coefficients $(h)$ are $11,175 \mathrm{~W} / \mathrm{m}^{2} \mathrm{~K}$ for the tubes and $22,012 \mathrm{~W} / \mathrm{m}^{2} \mathrm{~K}$ for the FW. The values are obtained using the Dittus-Boelter correlation taking into account the design water velocities of both circuits. Electrically insulated boundary conditions $\left(\partial_{n} \phi=0\right)$ are applied in these surfaces as well. In the inlet channel, the PbLi enters at a constant temperature $T=650 \mathrm{~K}$ and with a constant velocity $U_{0}=0.2 \mathrm{~mm} / \mathrm{s}$. 


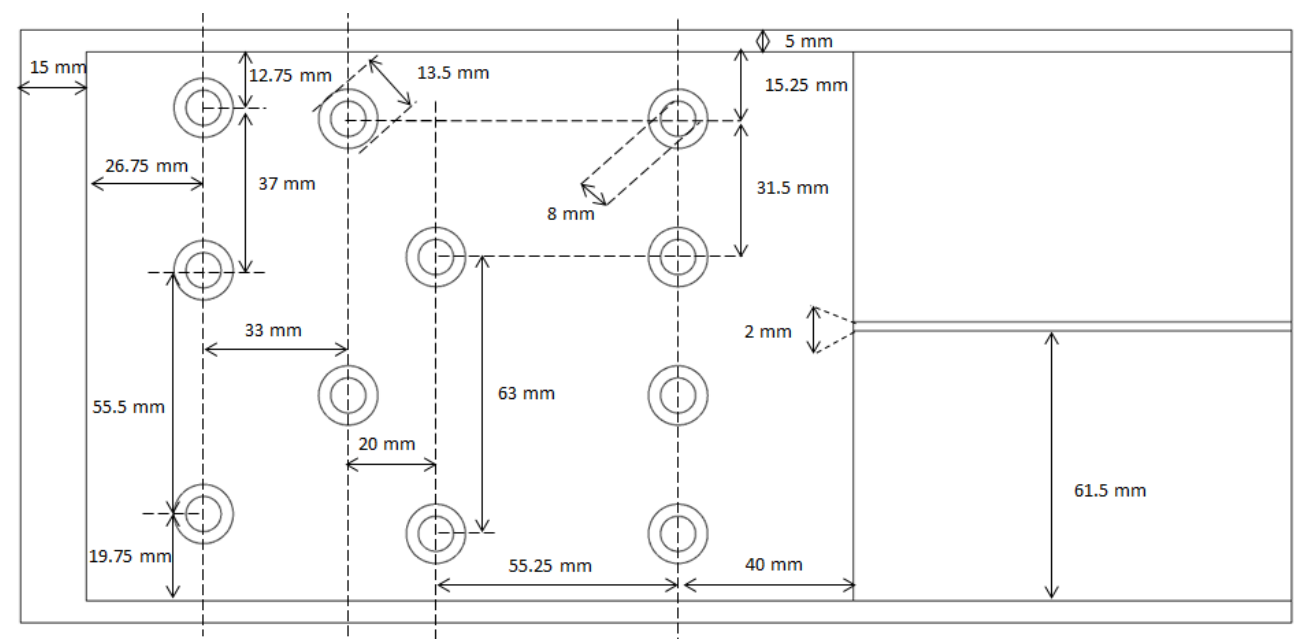

Figure 4. Geometrical input data.

Periodic boundary conditions are applied in the top and bottom steel plates to reproduce the presence of an analogous WCLL cell at the top and at the bottom of the studied one (translational symmetry). Likewise, periodic boundary conditions are applied in the lateral radial-poloidal surfaces since the PbLi parallel circuits stacked along the toroidal direction are supposed to be thermally similar and circulations between parallel circuits are expected to be negligible.

In the internal $\mathrm{PbLi} /$ steel surfaces, continuity of the temperature, heat flux, electric potential and electric currents are considered.

Concerning the initial conditions, a pure conductive heat transfer model (treating the $\mathrm{PbLi}$ as a solid material) has been used to obtain an initial temperature map. Using this map as the initial condition reduces the time needed to reach relevant conditions, which reduces significantly the computational time. This map is exposed in Figure 5. In agreement with previous studies [21], the hottest region is located at the end of the radial channels while the cooling tubes keep the frontal region at moderate temperatures $(600-700 \mathrm{~K})$.
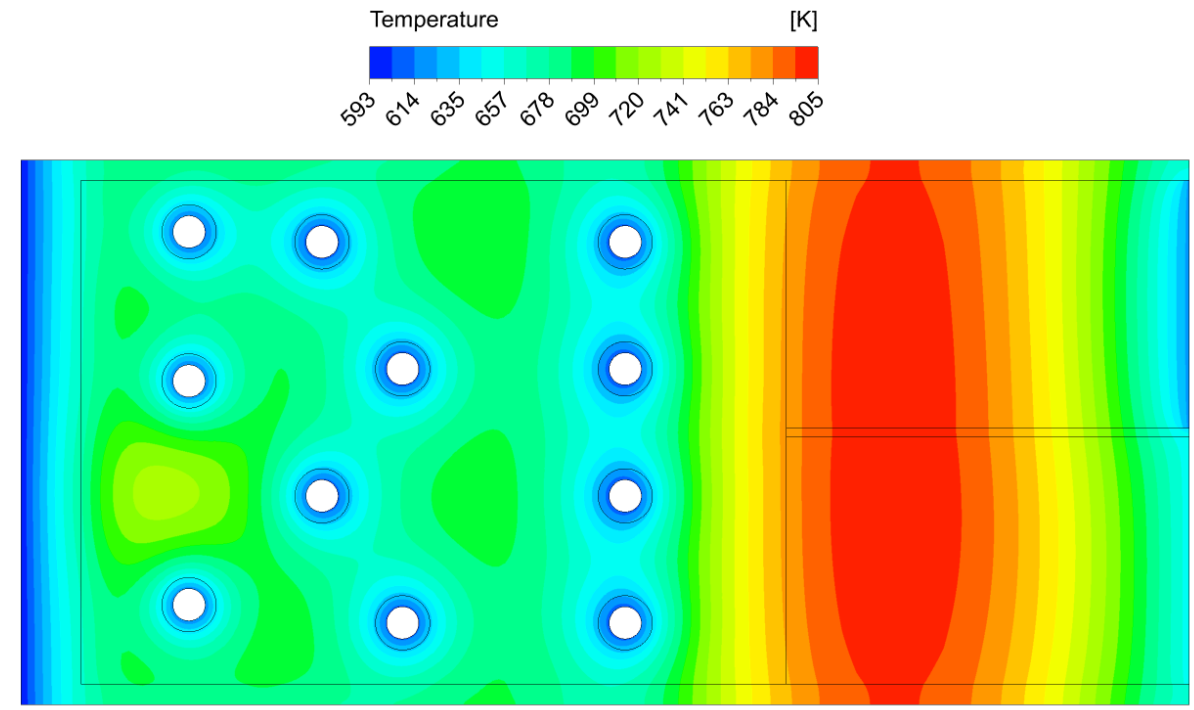

Figure 5. Initial temperature field obtained with a pure conductive model.

As mentioned in Section 1, the geometry has been simplified in order to employ a structured mesh. A multi-block structured mesh has been designed for this purpose using ICEM-CFD. O-grid structures are included in the vicinity of the tubes. After some testing, it was preferred to extend the radial elements of the tube walls towards the fluid domain 
in order to ensure good resolution in the boundary layers close to the tubes. For the same reasons, a hyperbolic clustering of cells has been applied towards the walls. Figure 6 depicts a zoomed view of the computational mesh used for the $\mathrm{Ha}=1000$ case.

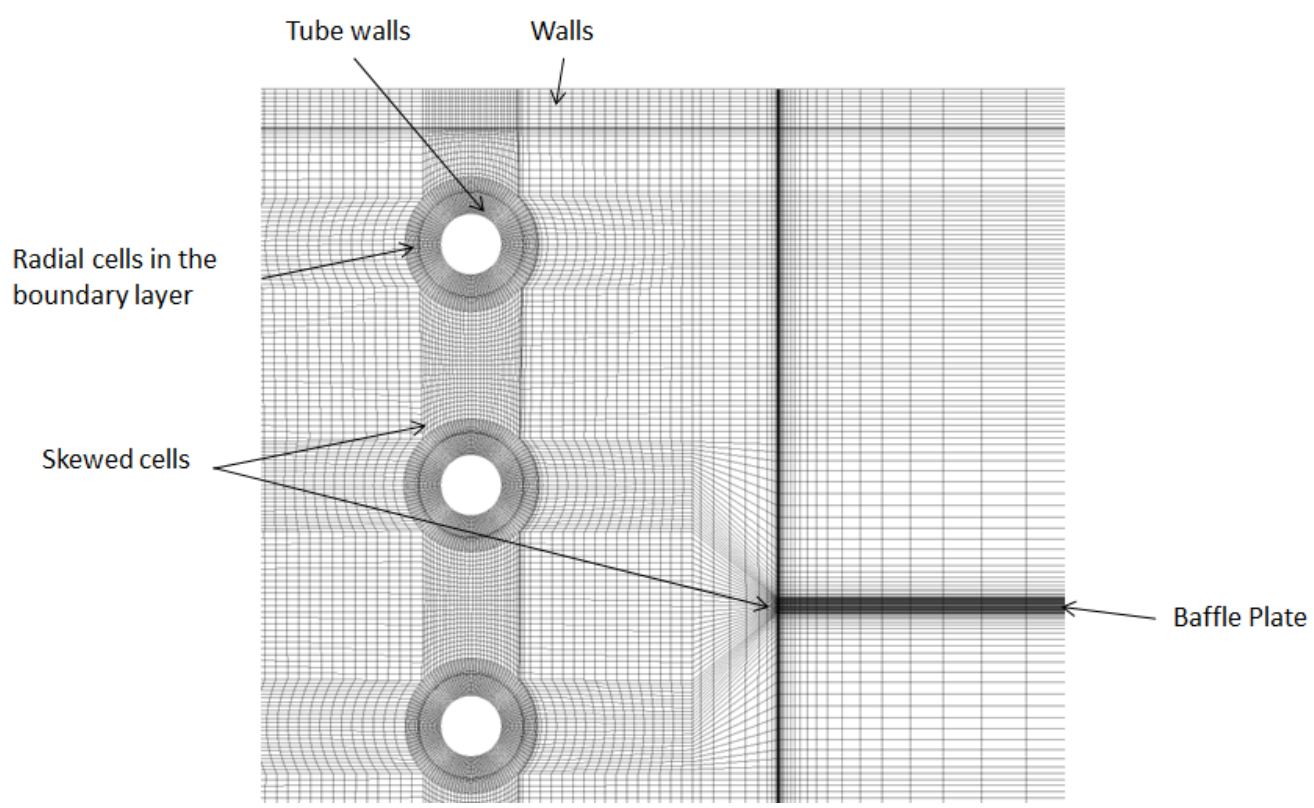

Figure 6. Detailed view of the computational mesh used for the $H a=1000$ case.

\section{Computational Results}

Transient magneto-convective simulations have been performed using the MHD solver integrated in the ANSYS-Fluent platform. This solver has been tested against the experimental results in a NaK loop [22] under pure MHD conditions. Moreover, a benchmarking exercise with another 4 MHD codes was successfully conducted under magneto-convective conditions [23].

Results are presented for the $H a=1000$ case and for the $H a=2000$ case after $200 \mathrm{~s}$ and $100 \mathrm{~s}$, respectively. The gravity field has been scaled according with (10). In both cases, the 3D solutions obtained present a quite good symmetry along the toroidal direction. This is expected not only because of the toroidal symmetry of the geometry but also because the magnetic field tends to align the convective vortexes along its direction. This kind of behavior is called quasi-two-dimensional (Q2D) turbulence [24]. For simplicity, results are presented only in the central radial-poloidal plane. Small deviation from these results can be found in other radial-poloidal planes but they are not significant. Weak temperature toroidal dependence has been found as well in hydrodynamics works in the regions where the tubes are toroidally oriented [21,25].

Figure 7 depicts a comparison between the temperature distribution of the purely conducting model (or the initial condition) and the results for $\mathrm{Ha}=1000$ and $\mathrm{Ha}=2000$ cases in the frontal part of the domain. Convective heat-transfer distorts the conductive temperature map. However, the effect is quite moderate which points to a heat transfer scenario dominated by conduction.

In any case, the flow motion boosts heat transfer near the cooling tubes decreasing peak and average temperatures. High temperature regions are slightly displaced towards the upper part of the domain due to the buoyancy force. There are relatively small but appreciable differences between both magneto convective cases. This indicates that even when keeping the overall ratio between buoyancy and Lorentz forces, local effects play a role on heat transfer. For example, higher velocity jets are developed next to the conducting walls at higher $\mathrm{Ha}$ numbers. 


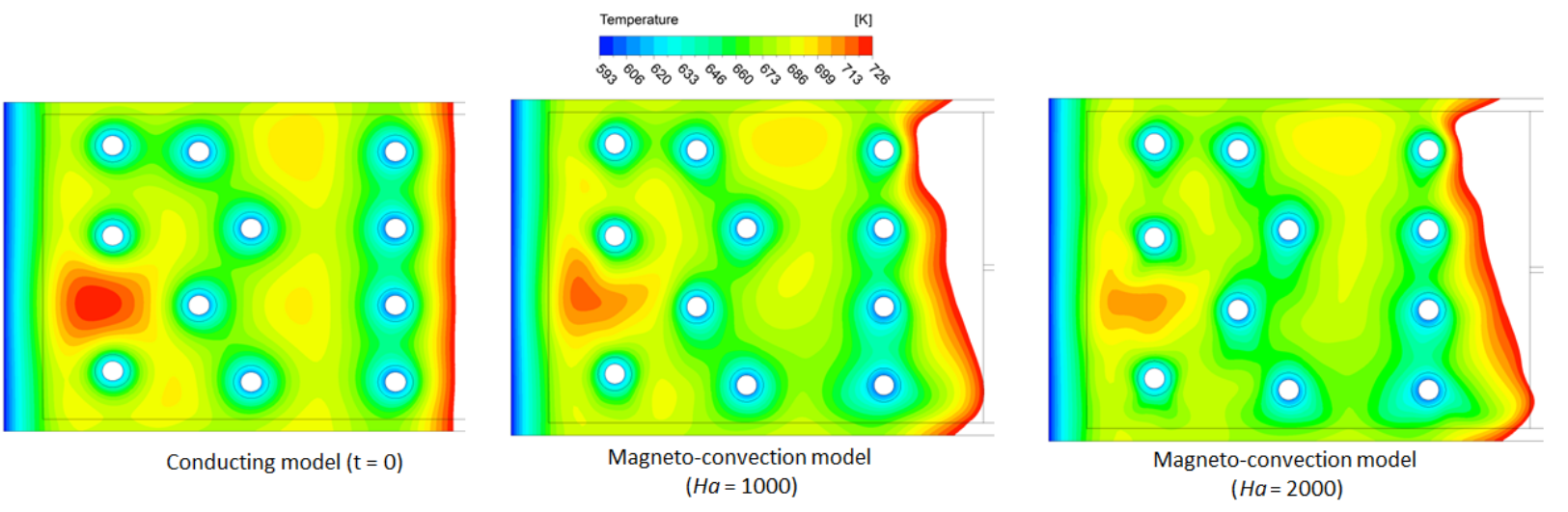

Figure 7. Comparison between the temperature distribution of the conducting and the magneto-convection model close to the FW.

The computed velocity vector field is exposed in Figure 8. In the first and second row of tubes, medium sized vortexes, (size comparable with the tube diameter) appear at both sides of each tube. Similar structures also appear next to the FW as a result of being the only cooled wall of the system. The vector field is qualitatively rather similar in both cases. Quantitatively, the velocity scale is of the order of $\sim 10^{-3} \mathrm{~m} / \mathrm{s}\left(\operatorname{Re} \sim 10^{2}\right)$ in both cases as well. However, peak velocities are higher (factor 2) in the $H a=2000$ case as a result of the electrical interaction between the fluid and conducting tube walls.

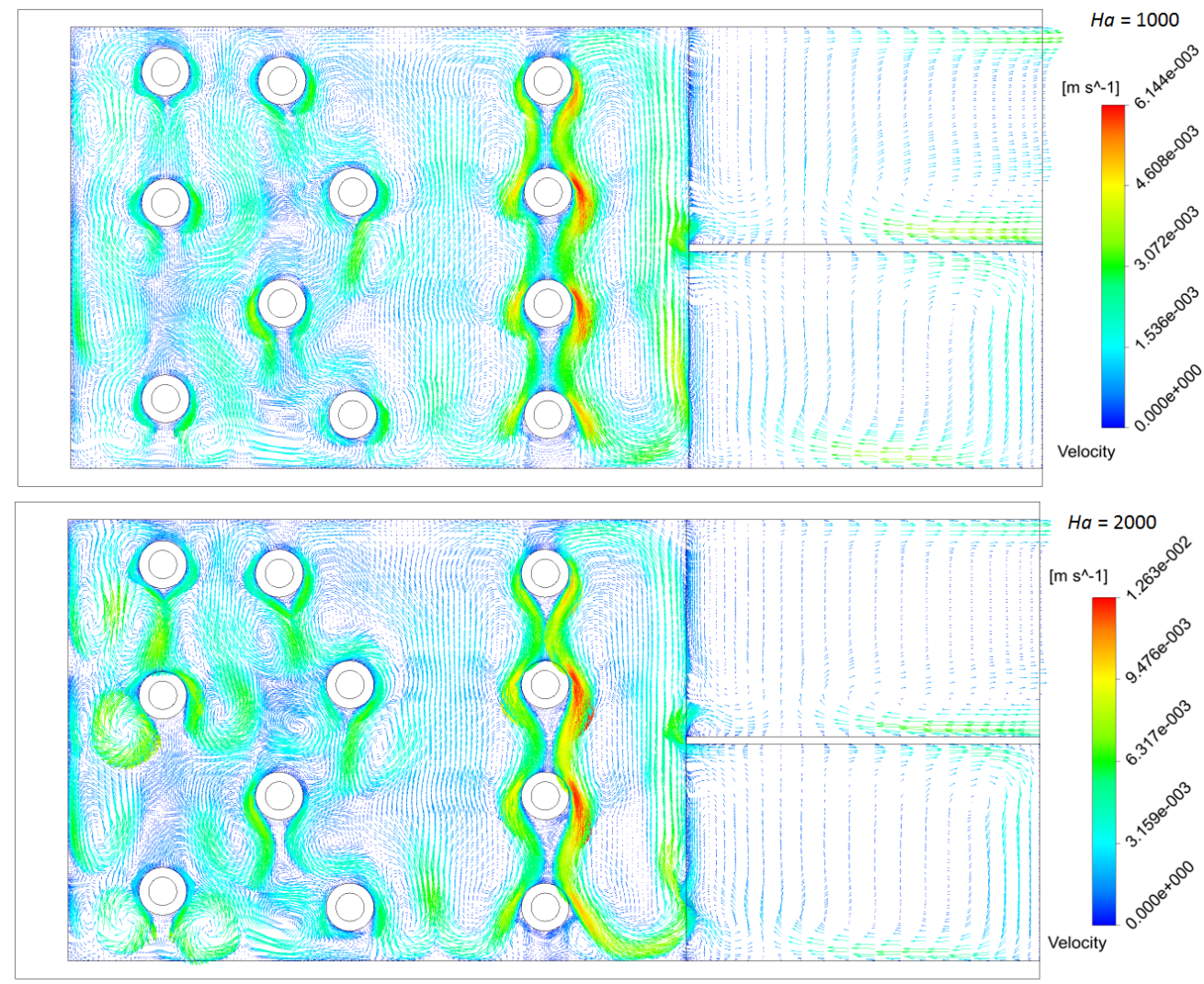

Figure 8. Velocity vector field in the central radial-poloidal plane for the $H a=1000$ case (top) and $H a=2000$ case (bottom).

Results also show a weak connection between the pressure driven flow in the radial channels and the rest of the domain. Indeed, a big recirculation region is developed in between the third row of tubes and the radial channels blocking the flow. As a result, the majority of the flow that comes from the inlet channel goes to the outlet channel 
through a narrow region close to the tip of the baffle plate. This effect was observed in pure MHD flows in simpler geometries that consider no tubes [26]. Recirculations regions are also observed in both the inlet and outlet radial channels. To confirm their apparation, the recirculation in the radial channels needs to be further investigated. In the present analyses, the proximity of the $\mathrm{PbLi}$ inlet and outlet faces might be introducing unrealistic effects. Other studies of the WCLL [17] predict recirculations in the radial channels as well. However, those seem to be less pronounced. The dissimilarities might be caused by differences in the heat source or the geometrical approximations employed in both works. Recirculations in the radial channels have been also computed for the HCLL TBM [16]. Nevertheless, the latter work considers a natural magneto-convection flow influenced by the HCLL horizontal cooling plates. These conditions are significantly different than in the WCLL radial channels.

Figure 9 depicts the electric potential distribution in both cases. Electric potential differences arises at both sides of the tubes. Comparing the potential contours with the vector velocity field, it is observed that local maxima and minima of potential are located in the center of the vortical structures. The vortexes extend along the whole toroidal direction of the computational domain. The alignment of the vortexes with the magnetic field direction is a characteristic of Q2D flows. In this flows, the contours of the electric potential coincide approximately with the stream lines of the flow. Indeed, assuming that the flow can be described in 2D, the electric potential is proportional to the stream function $(\psi)$ :

$$
\nabla_{2 D}^{2} \phi=\nabla_{2 D}\left(\vec{u} \times \vec{B}_{0}\right)=B_{0}\left(\partial_{x} u_{y}-\partial_{y} u_{x}\right)=-B_{0} \nabla_{2 D}^{2} \psi
$$

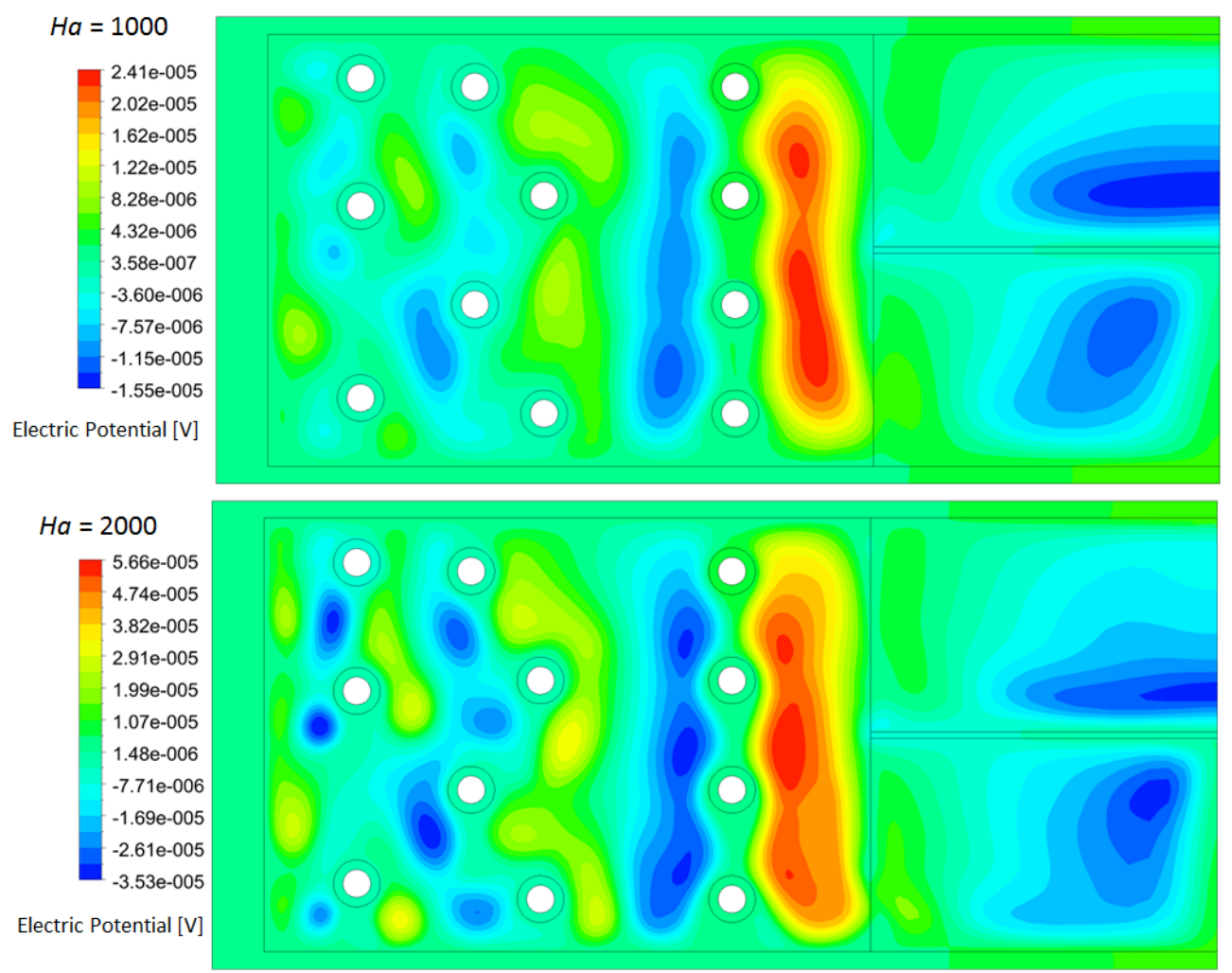

Figure 9. Electric potential in the central radial-poloidal plane for the $H a=1000$ case (top) and $H a=2000$ case (bottom).

3D potential iso-surfaces are exposed in Figure 10. The iso-surfaces are a good representation of the Q2D flow whose vortical structures are elongated along the magnetic field direction. This behavior is allowed and enhanced by symmetry of the geometry along this direction. This kind of elongated potential isosurfaces and their relation with the velocity 
stream-lines have been also obtained for the former EU-HCLL TBM conditions where the cooling of the $\mathrm{PbLi}$ was made by some radial-toroidal cooling plates [16].

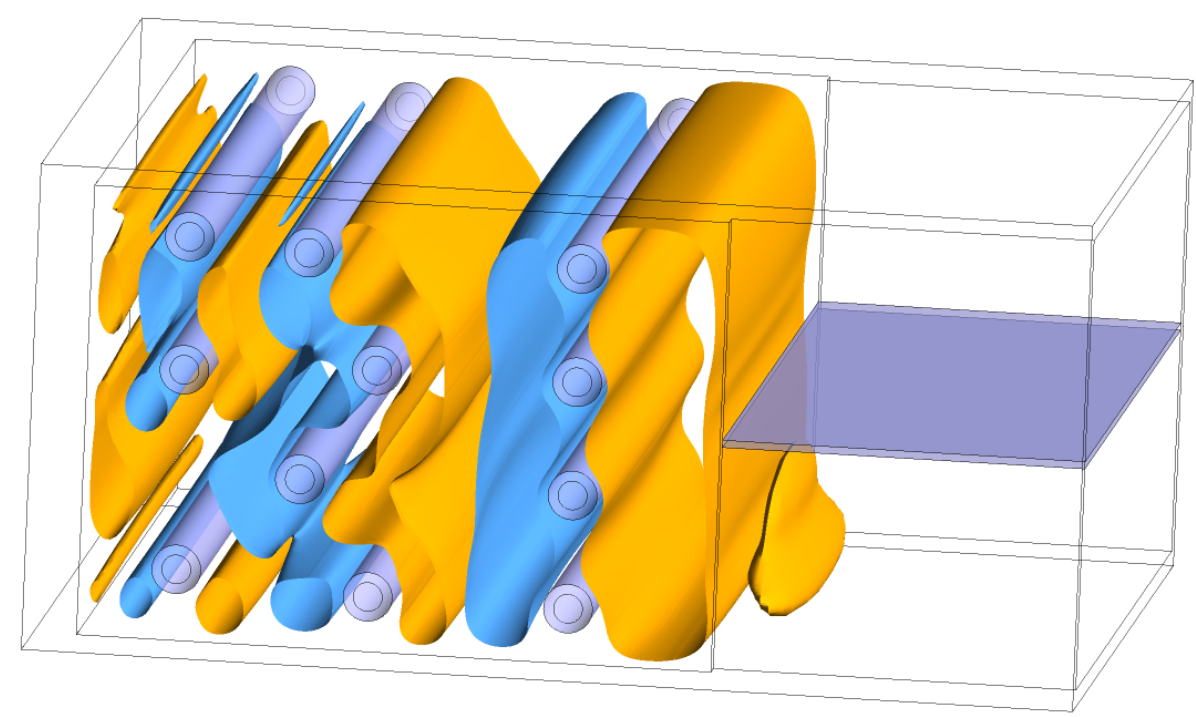

Figure 10. Isosurfaces of the electric potential $\left( \pm 10^{-5} \mathrm{~V}\right)$.

The sizes of the potential isosurfaces are dependent on the ratio $\frac{\mathrm{Gr}}{\mathrm{Ha}^{2}}$. Increasing the magnetic field while keeping $\mathrm{Gr}$ constant is expected to reduce the size of the vortexes [11].

\section{Estimation of the Nusselt Number}

Estimations of the Nusselt number $(\mathrm{N} u)$ are of great interest for design purposes. Knowing $\mathrm{Nu}$ or the heat transfer coefficient $h$ that appears in its definition allows performing system level heat transfer analyses. $\mathrm{Nu}$ represents the ratio between the convective and conductive processes in the fluid-solid interface:

$$
N u:=\frac{h \cdot L}{\kappa}
$$

The heat transfer coefficient can be estimated from the computational results by evaluating the average heat flux at the surface $\left(q_{s}\left[\mathrm{~W} / \mathrm{m}^{2}\right]\right)$ and the superficial temperature $\left(T_{s}\right)$ of each tube (and the FW) of the system:

$$
h=\frac{q_{s}}{T_{s}-T_{\infty}}
$$

The temperature $T_{\infty}$ is defined as a temperature sufficiently far away from the interface. Before computing, it is always necessary to specify the way in which the characteristic length $(L)$ and the temperature far away $\left(T_{\infty}\right)$ have been defined. In this case, $T_{\infty}$ has been defined as the average temperature of a cylindrical surface (a plane in the case of the FW) at a distance $2 L$ from the tube of interest. The tube surface and the cylindrical surface form a hollow cylinder (a prism in the case of the FW). The characteristic length is defined as the ratio between the volume and the area of external surfaces $(L=V / S)$.

The distance between the tube and cylindrical surface $(2 L)$ has been picked using the purely conducting model. Indeed, it is defined as the minimum length needed to obtain $N u=1$ with the conductive model results. This way, the definition is consistent with the extreme case of purely conductive heat transfer scenario. As a consequence of this definition, the characteristic length is slightly different for each tube but it is around $10 \mathrm{~mm}$ in every case which is roughly half the distance between tubes.

Table 2 depicts the heat transfer coefficients and $\mathrm{Nu}$ computed from the simulations results. The tubes of the different rows are labeled from the top to the bottom of the WCLL 
cell. This way the tube 1 of the row 1 is the tube located in the top left corner of Figure 4 and tube 4 of row 3 the one located in the bottom right corner.

Table 2. Average heat transfer coefficient and $N u$ in the fluid-solid interfaces.

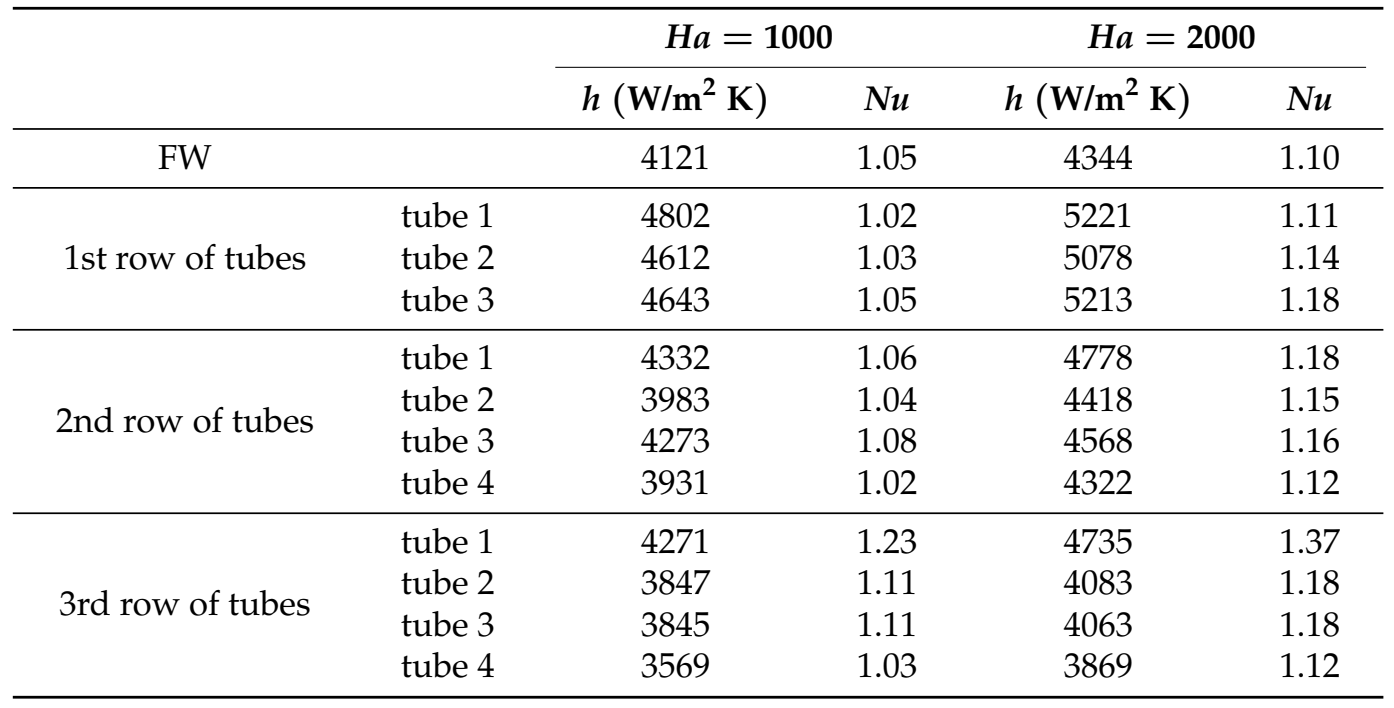

In agreement with the qualitative conclusions deduced from the obtained temperature maps (Figure 7), the quantitative analyses confirm that heat transfer is mostly ruled by conduction in the studied region. Indeed, $N u$ is very close to unity for all interfaces. There are small differences between the tubes and it is clear that the third row of tubes is slightly more affected by convection than the others. The third row of tubes is closer to the radial channels and therefore it is more influenced by the PbLi flow coming from them. In the other tubes, the flow is essentially moving by natural or free convection and unaffected by the flow of the channels.

It can also be deduced that $\mathrm{Nu}$ is around $5-10 \%$ higher when increasing $\mathrm{Ha}$ (and $\mathrm{Gr}$ ). This small difference is related with the increase of the velocity jets in the vicinity of the conducting walls with $\mathrm{Ha}$. In the real blanket conditions ( $\mathrm{Ha} \sim 8800$ and the real gravity field) the heat transfer by convection might play a more important role but it is not expected to be comparable with the conducting mechanism.

\section{Estimation of the Grashof Number}

The value of the Grashof number is dependent on the characteristic length $(b)$ and temperature differences $(\Delta T)$ picked in the definition (8). In the case of study this definition is not trivial since there are multiple heat sinks (each tube and the cooled FW) and the heat source is extended along the whole domain in a non-homogeneous way.

A conservative approach is considering the maximum temperature difference inside the $\mathrm{PbLi}$ (approximately $180 \mathrm{~K}$ ) and the total radial length of the frontal cavity as characteristic length. With this strategy it is obtained that $G r_{r}=1.59 \times 10^{9}$ in the $H a=2000$ case and $\mathrm{Gr}=2.93 \times 10^{10}$ in WCLL conditions. If the system were ruled by pure natural convection heat transfer, the Reynolds number would be $R e=\frac{G r}{H a^{2}}=4 \times 10^{2}$ which is of the order of the average Re obtained in the simulations.

Alternatively, a local definition can be used based on the temperature differences between some previously defined toroidal-poloidal planes located at different radial positions. This definition is motivated by tubes disposition in rows and by the radial dependence of the heat source.

The planes are defined in the middle of each tube row and in between them. This means that distances between two adjacent planes are approximately the size of the Q2D vortexes obtained in the simulations. Multiple values of $G r$ have been derived considering 
the differences between the planes average temperatures $(\Delta T)$ and the distance $(b)$ between them. The $G r$ computed this way are presented in Figure 11 for the $H a=2000$ case.

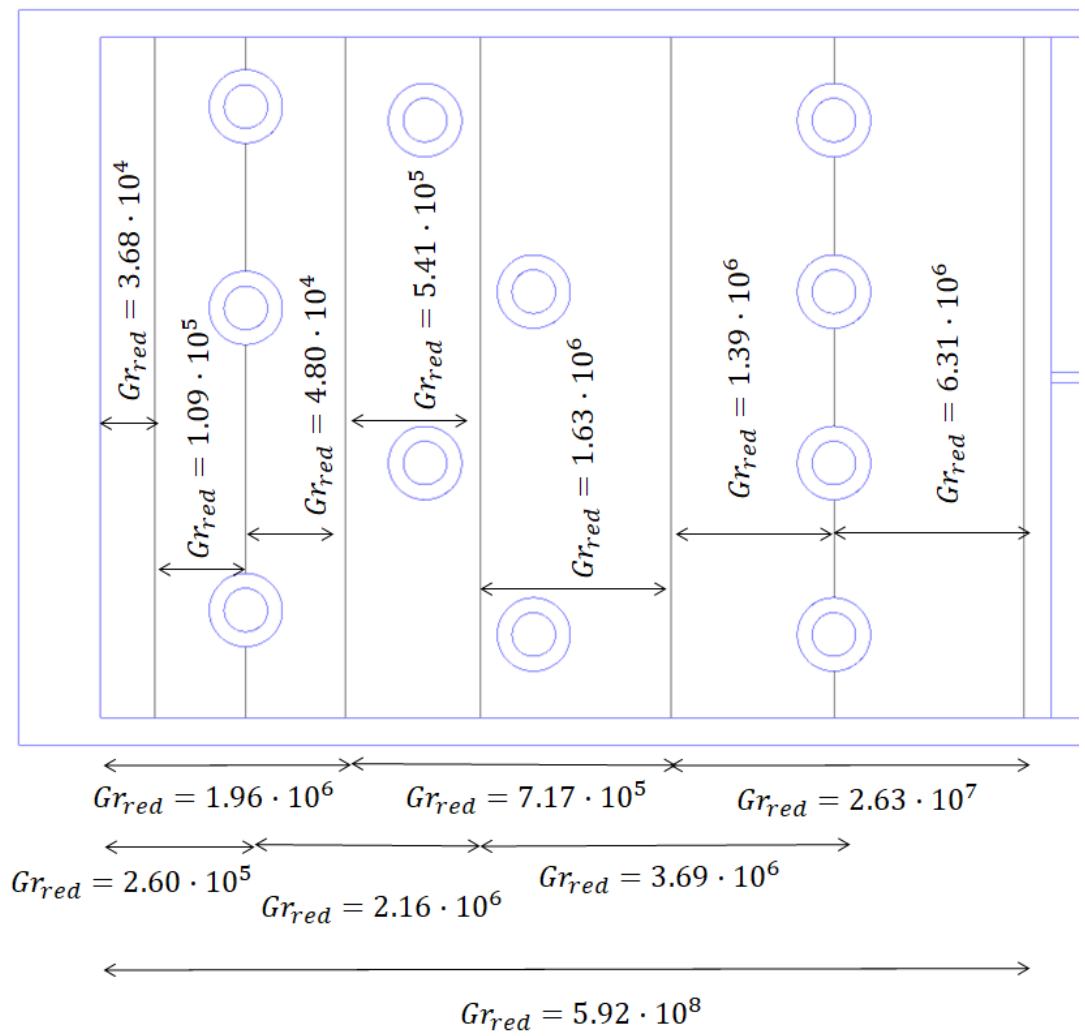

Figure 11. Reduced Grashof number for different toroidal-poloidal planes.

The values presented are calculated for a reduced gravity field (10). For obtaining the results in the real WCLL conditions, $G r$ has to be multiplied by the square of the ratio between target $\mathrm{Ha}(8800)$ and the reduced $\mathrm{Ha}$ (2000), in other words $4.4^{2}$. After the rescaling, the values obtained in the real conditions vary between $10^{5}$ and $10^{10}$, depending on the planes considered. Most likely, the most relevant $\mathrm{Gr}$ are the ones computed using two adjacent planes since this is the size of the convective vortexes associated to the problem. This implies a maximum value of $1.22 \times 10^{8}$ in between the third row of tubes and the radial channels and a minimum value of $7.13 \times 10^{5}$ in between the FW plane and the first plane. It is worth noting that the $G r$ obtained in between two rows of tubes is of the order of $10^{7}$ which is one order of magnitude below the initial estimations.

\section{Conclusions}

This work presents a magneto-convective simulation of the EU-WCLL blanket central outboard elementary cell. The analyses are focused on the frontal region which is close to the FW and subjected to a very high volumetric heat source and to a very high magnetic field. Geometrically, this region is characterized by a group of cooling tubes that crosses the fluid domain mostly in the perpendicular direction to the flow (parallel to the magnetic field). Simulations have been implemented using the MHD solver of ANSYS-Fluent.

According to the results, the dynamics of this region is driven mostly by natural convection. Little influence of the colder radial $\mathrm{PbLi}$ flow was found in the frontal region. Indeed, most of the pressure driven flow goes from the inlet to the outlet channel very close to the tip of the baffle plate interacting weakly with the rest of the domain. Medium size vortexes (dimension of the order of the tube diameter) appear in the frontal part of the domain. Having vortexes isolated in the frontal region can have important implications for tritium transport since high concentrations can arise in these regions. 
Heat transfer is mostly driven by conduction in the system. Indeed, temperature profiles are relatively similar to the purely conductive solution used as an initial condition. Hot spots are a bit distorted and displaced towards the top part of the domain but not in a strong way. This effect is a consequence of the magnetic field that suppresses the heat transfer via advection. The suppression of the advective heat transfer mechanism by the magnetic field was an expected result in agreement with previous experiences. Despite the conductive nature of the heat transfer, the dense network of water tubes are able to keep the PbLi and more importantly the structural steel below critical temperatures $\left(550^{\circ} \mathrm{C}\right)$.

$\mathrm{Nu}$ numbers and heat transfer coefficients have been computed for each cooling tube and for the FW. Values very close to unity have been obtained for $\mathrm{Nu}$ in every case. $\mathrm{Gr}$ numbers have been also estimated using the simulations outcomes. A global $\mathrm{Gr}$ of $\sim 2.93 \times 10^{10}$ has been obtained from the maximum temperature difference obtained in the WCLL cell. Local estimations of $G r$ in between rows of tubes ( $\sim$ size of the vortexes) provides a value two or three orders of magnitude smaller: $\mathrm{Gr} \sim 10^{7}, 10^{8}$.

Future developments should be focused on reaching the actual values of the magnetic field and gravity field in the blanket. Moreover, the influence of the tube curvature should be evaluated since they will break the toroidal symmetry and possibly the Q2D structures. In fact, it is expected that curvature of the third row of tubes will break the toroidal extension of the bigger vortexes next to the baffle plate. Moreover, some of this tubes penetrate in the PbLi radial channels which will mitigate the hot spot located next to the end of the baffle plate. The effects could significantly affect the recirculation found in the radial channels. This circumstance has to be further investigated.

To include the tube curvature, unstructured tetrahedral elements will be most likely unavoidable. An investigation of the numerical stability of magneto-convective problems with this kind of meshes should be performed. Finally, different orientations of the gravity and the magnetic field should be analyzed. This would allow studying other scenarios in WCLL cells different from the central one. These have variable orientations with respect to the horizontal plane. Besides, the poloidal component of the magnetic field might play also a significant role on magneto-convective results.

Author Contributions: Conceptualization, F.R.U. and I.F.-B.; methodology, F.R.U.; software, F.R.U.; formal analysis, F.R.U.; writing-original draft preparation, F.R.U.; writing-review and editing, I.F.-B. and D.R.; visualization, F.R.U. and I.F.-B.; supervision, I.F.-B. and D.R.; project administration, D.R.; funding acquisition, D.R. All authors have read and agreed to the published version of the manuscript.

Funding: This work has been carried out within the framework of the EUROfusion Consortium and has received funding from the Euratom research and training programme 2014-2018 and 2019-2020 under grant agreement No 633053. The views and opinions expressed herein do not necessarily reflect those of the European Commission.

Institutional Review Board Statement: Not applicable.

Informed Consent Statement: Not applicable.

Data Availability Statement: Not applicable.

Acknowledgments: This work was partially supported by the computing facilities of Extremadura Research Centre for Advanced Technologies (CETA-CIEMAT), funded by the European Regional Development Fund (ERDF). CETA-CIEMAT belongs to CIEMAT and the Government of Spain. The authors thank Alessandro Del Nevo and the rest of the WCLL design team for providing the CAD files, heat source functions and boundary values needed for this work.

Conflicts of Interest: The authors declare no conflict of interest. 


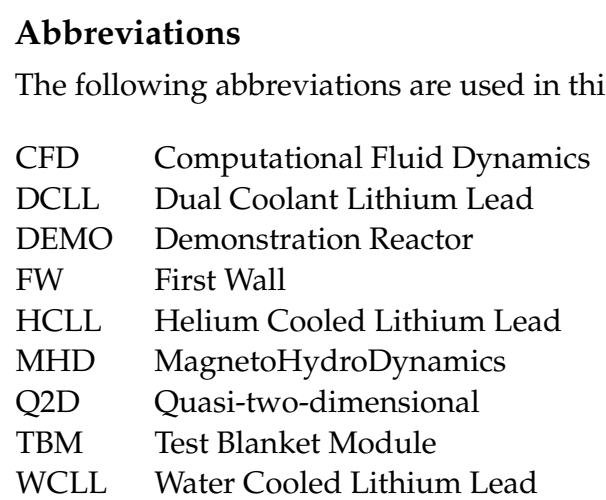

\section{References}

1. Boccaccini, L.; Aiello, G.; Aubert, J.; Bachmann, C.; Barrett, T.; Del Nevo, A.; Demange, D.; Forest, L.; Hernandez, F.; Norajitra, P.; et al. Objectives and status of EUROfusion DEMO blanket studies. Fusion Eng. Des. 2016, 109-111, 1199-1206. [CrossRef]

2. Federici, G.; Boccaccini, L.; Cismondi, F.; Gasparotto, M.; Poitevin, Y.; Ricapito, I. An overview of the EU breeding blanket design strategy as an integral part of the DEMO design effort. Fusion Eng. Des. 2019, 141, 30-42. [CrossRef]

3. Del Nevo, A.; Arena, P.; Caruso, G.; Chiovaro, P.; Di Maio, P.; Eboli, M.; Edemetti, F.; Forgione, N.; Forte, R.; Froio, A.; et al. Recent progress in developing a feasible and integrated conceptual design of the WCLL BB in EUROfusion project. Fusion Eng. Des. 2019, 146, 1805-1809. [CrossRef]

4. Moro, F.; Arena, P.; Catanzaro, I.; Colangeli, A.; Del Nevo, A.; Flammini, D.; Fonnesu, N.; Forte, R.; Imbriani, V.; Mariano, G.; et al. Nuclear performances of the water-cooled lithium lead DEMO reactor: Neutronic analysis on a fully heterogeneous model. Fusion Eng. Des. 2021, 168, 112514. [CrossRef]

5. Smolentsev, S.; Morley, N.B.; Abdou, M. Magnetohydrodynamic and Thermal Issues of the SiCf/SiC Flow Channel Insert. Fusion Sci. Technol. 2006, 50, 107-119. [CrossRef]

6. Urgorri, F.R.; Smolentsev, S.; Fernández-Berceruelo, I.; Rapisarda, D.; Palermo, I.; Ibarra, A. Magnetohydrodynamic and thermal analysis of PbLi flows in poloidal channels with flow channel insert for the EU-DCLL blanket. Nucl. Fusion 2018, 58, 106001. [CrossRef]

7. Bühler, L.; Mistrangelo, C. MHD flow and heat transfer in model geometries for WCLL blankets. Fusion Eng. Des. 2017, 124, 919-923. [CrossRef]

8. Mistrangelo, C.; Bühler, L. Numerical Study of Fundamental Magnetoconvection Phenomena in Electrically Conducting Ducts. IEEE Trans. Plasma Sci. 2012, 40, 584-589. [CrossRef]

9. Müller, U.; Bühler, L. Magnetohydrodynamics in Channels and Containers; Springer: Berlin/Heidelberg, Germany, 2001.

10. Zhang, X.; Zikanov, O. Mixed convection in a horizontal duct with bottom heating and strong transverse magnetic field. J. Fluid Mech. 2014, 757, 33-56. [CrossRef]

11. Smolentsev, S.; Vetcha, N.; Abdou, M. Effect of a magnetic field on stability and transitions in liquid breeder flows in a blanket. Fusion Eng. Des. 2013, 88, 607-610. [CrossRef]

12. Vetcha, N.; Smolentsev, S.; Abdou, M.; Moreau, R. Study of instabilities and quasi-two-dimensional turbulence in volumetrically heated magnetohydrodynamic flows in a vertical rectangular duct. Phys. Fluids 2013, 25, 024102. [CrossRef]

13. Vetcha, N.; Smolentsev, S.; Abdou, M. Theoretical Study of Mixed Convection in Poloidal Flows of DCLL Blanket. Fusion Sci. Technol. 2009, 56, 851-855. [CrossRef]

14. Vetcha, N.; Smolentsev, S.; Abdou, M. Stability Analysis for Buoyancy-Opposed Flows in Poloidal Ducts of the DCLL Blanket. Fusion Sci. Technol. 2011, 60, 518-522. [CrossRef]

15. Zikanov, O.; Listratov, Y.; Sviridov, V. Natural convection in horizontal pipe flow with a strong transverse magnetic field. J. Fluid Mech. 2013, 720, 486-516. [CrossRef]

16. Mistrangelo, C.; Bühler, L.; Aiello, G. Buoyant-MHD Flows in HCLL Blankets Caused by Spatially Varying Thermal Loads. IEEE Trans. Plasma Sci. 2014, 42, 1407-1412. [CrossRef]

17. Yan, Y.; Ying, A.; Abdou, M. Numerical study of magneto-convection flows in a complex prototypical liquid-metal fusion blanket geometry. Fusion Eng. Des. 2020, 159, 111688. [CrossRef]

18. Tassone, A.; Caruso, G.; Giannetti, F.; Del Nevo, A. MHD mixed convection flow in the WCLL: Heat transfer analysis and cooling system optimization. Fusion Eng. Des. 2019, 146, 809-813. [CrossRef]

19. Martelli, D.; Venturini, A.; Utili, M. Literature review of lead-lithium thermophysical properties. Fusion Eng. Des. 2019, 138, 183-195. [CrossRef]

20. Guillemot, F. Material Property Handbook Pilot Project on EUROFER97 (MTA EK, KIT); Technical Report EFDA-D-2MRP77; EUROfusion: Garching, Germany, 2016.

21. Edemetti, F.; Martelli, E.; Tassone, A.; Caruso, G.; Nevo, A.D. DEMO WCLL breeding zone cooling system design: Analysis and discussion. Fusion Eng. Des. 2019, 146, 2632-2638. [CrossRef] 
22. Satyamurthy, P.; Swain, P.; Tiwari, V.; Kirillov, I.; Obukhov, D.; Pertsev, D. Experiments and numerical MHD analysis of LLCB TBM Test-section with NaK at $1 \mathrm{~T}$ magnetic field. Fusion Eng. Des. 2015, 91, 44-51. [CrossRef]

23. Smolentsev, S.; Rhodes, T.; Yan, Y.; Tassone, A.; Mistrangelo, C.; Bühler, L.; Urgorri, F.R. Code-to-Code Comparison for a PbLi Mixed-Convection MHD Flow. Fusion Sci. Technol. 2020, 76, 653-669. [CrossRef]

24. Sommeria, J.; Moreau, R. Why, how, and when, MHD turbulence becomes two-dimensional. J. Fluid Mech. 1982, 118, 507-518. [CrossRef]

25. Edemetti, F.; Martelli, E.; Del Nevo, A.; Giannetti, F.; Arena, P.; Forte, R.; Di Maio, P.A.; Caruso, G. On the impact of the heat transfer modelling approach on the prediction of EU-DEMO WCLL breeding blanket thermal performances. Fusion Eng. Des. 2020, 161, 112051. [CrossRef]

26. Tassone, A.; Caruso, G.; Del Nevo, A.; Di Piazza, I. CFD simulation of the magnetohydrodynamic flow inside the WCLL breeding blanket module. Fusion Eng. Des. 2017, 124, 705-709. [CrossRef] 